\title{
Fair Resource Allocation with QoS Guarantee in Secure Multiuser TDMA Networks
}

\author{
Zhiquan Bai, ${ }^{1}$ Tongtong Wang, ${ }^{1}$ Piming Ma $\mathbb{D},{ }^{1}$ Yanbo $\mathrm{Ma}^{2}$ and Kyungsup Kwak $\mathbb{D}^{3}$ \\ ${ }^{1}$ Shandong Provincial Key Laboratory of Wireless Communication Technology, School of Information Science and \\ Engineering, Shandong University, Jinan 250100, China \\ ${ }^{2}$ School of Management Science and Engineering, Shandong University of Finance and Economics, Jinan 250014, China \\ ${ }^{3}$ Graduate School of Information and Telecommunications, INHA University, Incheon 402-751, Republic of Korea \\ Correspondence should be addressed to Piming Ma; mapiming@sdu.edu.cn
}

Received 6 January 2018; Revised 18 May 2018; Accepted 11 June 2018; Published 11 July 2018

Academic Editor: Giuseppe Piro

Copyright (C) 2018 Zhiquan Bai et al. This is an open access article distributed under the Creative Commons Attribution License, which permits unrestricted use, distribution, and reproduction in any medium, provided the original work is properly cited.

\begin{abstract}
We investigate a secure multiuser time division multiple access (TDMA) system with statistical delay quality of service (QoS) guarantee in terms of secure effective capacity. An optimal resource allocation policy is proposed to minimize the $\beta$-fair cost function of the average user power under the individual QoS constraint, which also balances the energy efficiency and fairness among the users. First, convex optimization problems associated with the resource allocation policy are formulated. Then, a subgradient iteration algorithm based on the Lagrangian duality theory and the dual decomposition theory is employed to approach the global optimal solutions. Furthermore, considering the practical channel conditions, we develop a stochastic subgradient iteration algorithm which is capable of dynamically learning the intended wireless channels and acquiring the global optimal solution. It is shown that the proposed optimal resource allocation policy depends on the delay QoS requirement and the channel conditions. The optimal policy can save more power and achieve the balance of the energy efficiency and the fairness compared with the other resource allocation policies.
\end{abstract}

\section{Introduction}

Due to the broadcast nature of wireless communications, much more attention has been paid to the issues of privacy and security in wireless communication networks. Traditionally, security is achieved by cryptographic encryption protocols of the upper layers. However, the security of encryption will be invalid if the wiretappers have huge computational power. From the information-theoretic perspective, physicallayer security can guarantee the reliable secure transmission via utilizing the physical characteristics of wireless channels. The concept of information-theoretic secrecy was originally introduced by Shannon [1]. Then, a relaxed notion of secrecy was presented by Wyner in his seminal work [2] based on the concept of a wiretap channel model. It is shown that the transmitter can securely transmit the messages to the receiver with a non-zero rate (called secrecy rate) if the receiver enjoys better channel conditions than the eavesdropper. The results were subsequently extended to the broadcast channels [3] and Gaussian channel [4], respectively. Various physical-layer techniques, such as the use of interference or artificial noise to confuse the eavesdropper, the multi-antenna, beamforming, and resource allocation techniques, were proposed in [5-8] to improve the secrecy capacity, also known as the largest secrecy rate.

In recent years, a large amount of work has been devoted to the resource allocation based on physical-layer security. In [9-12], resource allocation schemes of OFDM secrecy systems were studied in the case of single-user system, two-user system, and multiuser system, respectively. Specifically, joint power and subcarrier allocation were investigated for the network with the coexistence of secure users and normal users [11]. Furthermore, a joint subcarrier and power allocation algorithm with artificial noise was designed in [12] to improve the security of the OFDM wiretap channels. In addition, the secure resource allocation was widely investigated in relay networks. Reference [13] considered an amplify-and-forward (AF) relay-aided secure multicarrier communication system 
and solved the resource allocation problem to maximize the sum secrecy rate. On the other hand, J. Huang proposed the cooperative jamming strategies for two-hop relay networks and investigated the optimization problem of maximizing the secrecy rate with certain power constraints and minimizing the transmit power with a fixed secrecy rate [14]. Moreover, large-scale multiple-input multiple-output (MIMO) technique was exploited by the relay system in the presence of a passive eavesdropper and an energy-efficient power allocation scheme was proposed in [15]. Recently, some researchers also focused on the secure resource allocation problem of the cognitive radio networks (CRNs) [16]. In [17], instantaneous and ergodic resource allocation problems were investigated in CRNs with guaranteed secrecy rate of the primary users. Secure robust resource allocation was taken into account for the relay-assisted CRNs in [18]. Different from the above two literatures, [19] proposed the power allocation policy for the physical-layer security in cognitive relay networks from the perspective of auction theory. Device-to-Device (D2D) communications have also been proposed recently to improve the spectral efficiency. Physical-layer security in D2D communications was studied in [20-22] and the corresponding resource allocation schemes were proposed.

It is worth mentioning that the framework employed above is not suitable for the delay-sensitive multimedia applications since Shannon theory places no restriction on the delay of the transmission scheme. However, delay-tolerant guarantee as one of the essential QoS merits plays a pivotal important role in the secure communications. Furthermore, deterministic delay bound QoS is commonly not available in wireless networks due to the unpredictable nature of the wireless fading channels. Based on the effective capacity theory proposed by $\mathrm{Wu}$ and Negi in [23, 24], our preliminary work has investigated the delay QoS guarantee for secrecy system [25], which took the secure effective capacity as the QoS metric. Reference [26] also discussed the security problem under the QoS constraints for CR system with similar method. Secure effective capacity framework based on the effective capacity theory is employed as a bridge for the cross-layer model, where the queue at the data link layer can be served by the resource allocation scheme of the physical layer. It is a convenient approach to investigate the QoS support mechanisms for wireless secure communications.

As the extended application of the secure effective capacity for secure wireless networks, we propose a cross-layer model based power and time slot allocation policy for wiretap time division multiple access (TDMA) systems, where the delay-tolerant requirement is considered. Regarding the existence of multiple users in the system, a class of so-called $\beta$-fair cost functions was introduced to balance the energy efficiency and the fairness [27]. First, we discuss the issue of minimizing a general cost function of the average user power subjected to the delay QoS constraint of the individual user. A dual-based iterative algorithm based on the Lagrangian dual technique $[28,29]$ and the dual decomposition theory $[30,31]$ is employed to solve the optimization problem. In addition, according to the stochastic optimization theory, we develop a stochastic subgradient iterative algorithm with unknown cumulative distribution function (CDF) of the fading channel, which can dynamically learn the underlying channel distribution and approach the optimal solution. Then, we also provide the non-delayed policy without considering the delay QoS requirement for comparison.

To summarize, the main contributions of this work are as follows.

(1) The secure effective capacity based on the cross-layer framework is employed for the wiretap TDMA system.

(2) Based on the cross-layer framework, two resource allocation schemes are addressed, the optimal resource allocation policy, and the non-delayed resource allocation policy. The optimal policy adaptively allocates the power and time slot to minimize the utility functions of the average user power subjected to the delay QoS requirement. The nondelayed policy minimizes the objective function without considering the delay QoS guarantee.

(3) The optimization problem is solved and analyzed based on the Lagrangian duality theory and the stochastic optimization theory. The optimal policy can get better energy efficiency and also achieve the balance of the energy efficiency and the fairness.

The rest of the paper is organized as follows. Section 2 describes the system model and the delay QoS guarantee. The optimal resource allocation algorithm and the non-delayed resource allocation policy are proposed in Sections 3 and 4 , respectively. Section 5 provides the numerical results to illustrate the performance of the proposed resource allocation schemes. Finally, conclusions and discussions are drawn in Section 6.

\section{System Overview and Delay QoS Guarantee}

In this section, we first present the system model of the wiretap TDMA system. Then, the issues of the statistical delay QoS guarantee are addressed based on the secure effective capacity.

2.1. System Model. In this paper, we investigate a network consisting of multiple users, one legitimate receiver, and one eavesdropper. $L$ users communicate with the legitimate receiver in the presence of an eavesdropper, which is shown in Figure 1. All the users are equipped with single antenna, and the solid and dash lines represent the main and the wiretap links, respectively. We adopt the TDMA scheme as the multiple access scheme to eliminate the multiuser interference in the system and the channel is shared by multiple users at different time slots. For user $l$, let $h_{l}$ and $g_{l}$ denote the main and the wiretap channel power gains, respectively. We assume a discrete-time block-fading channel model such that $h_{l}$ and $g_{l}$ remain constant within a frame duration $T_{f}$ but experience a jointly stationary and ergodic fading process from one frame to another with continuously joint known probability density function (PDF). Then, at time slot $n$, the received signals at the legitimate receiver and the eavesdropper from the transmit user $l$ are given by

$$
\begin{aligned}
& y_{l}(n)=\sqrt{h_{l}(n)} x_{l}(n)+a_{l}(n), \\
& z_{l}(n)=\sqrt{g_{l}(n)} x_{l}(n)+b_{l}(n),
\end{aligned}
$$




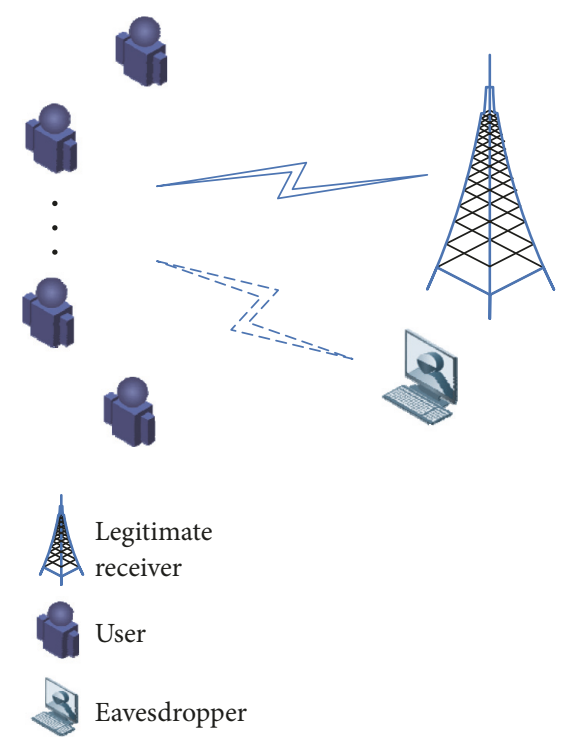

FIGURE 1: System model of the multiuser TDMA secrecy networks.

respectively, where $x_{l}(n)$ represents the transmitted signal, and $a_{l}(n)$ and $b_{l}(n)$ denote the corresponding additive white Gaussian noise (AWGN) with zero-mean and unit variance at the the legitimate receiver and the eavesdropper, respectively. Like $[5,7]$, we suppose that all the users have full knowledge of both the main channel state information (CSI) and the wiretap CSI which are described by the vectors $\mathbf{h}$ := $\left[h_{1}, \ldots, h_{L}\right]^{T}$ and $\mathbf{g}:=\left[g_{1}, \ldots, g_{L}\right]^{T}$, respectively, where $[\cdot]^{T}$ denotes the transposition. Herein, we neglect the time reference for simplicity. We assume that each time slot is shared by multiple users over non-overlapping and dedicated time fractions $\left\{\tau_{l}\right\}_{l=1}^{L}$. Without loss of generality, each frame duration is supposed to be normalized as $T_{f}=1$ and we have $\sum_{l=1}^{L} \tau_{l} \leq 1$. We further assume that the users allocated no transmission time slots will not be allocated any power. Given $\tau_{l}$ and the corresponding transmit power $p_{l}$, the instantaneous secrecy rate of the $l$-th user is given by $[32,33]$ as follows:

$$
\begin{aligned}
& r_{\text {sec }}\left(\tau_{l}, p_{l}\right) \\
& = \begin{cases}\tau_{l}\left[\log _{2}\left(1+\frac{h_{l} p_{l}}{\tau_{l}}\right)-\log _{2}\left(1+\frac{g_{l} p_{l}}{\tau_{l}}\right)\right], & \tau_{l}>0, h_{l}>g_{l} \\
0, & \text { else }\end{cases}
\end{aligned}
$$

which shows that the secrecy can be achieved when the main channel is better than the eavesdropper channel; i.e., $h_{l}>g_{l}$.

2.2. Delay QoS Guarantee. In this subsection, we utilize the secure effective capacity to measure the throughput of the secrecy system under QoS constraint. The effective capacity characterizes the maximum constant arrival rate that the channel can support and guarantees a given delay QoS requirement.

The information source stream from the upper layers and the service process driven by the resource control scheme at the physical layer are matched via the queue at the data link layer. It is assumed that the source message stream has a specified delay bound $D_{\max }[23,24]$ and the violation probability of the delay bound should not be larger than a certain nonnegative value $\varepsilon$ as

$$
\operatorname{Pr}\left\{D(\infty)>D_{\max }\right\} \leq \varepsilon,
$$

where $D(\infty)$ is the delay experienced by a source message stream in a steady state. Then, we mark the QoS requirement of the source message stream as $\left\{D_{\max }, \varepsilon\right\}$. As shown in [24], the effective capacity is related to the theory of large deviations. The probability of $D(\infty)$ exceeding the delay bound $D_{\max }$ can be expressed as follows:

$$
\operatorname{Pr}\left\{D>D_{\max }\right\} \approx e^{-\theta^{*} E_{B} D_{\max }},
$$

where $E_{B}$ is defined as the effective bandwidth of the source message stream, $\theta^{*}$ is a value obtained by solving the function $E_{C}(\theta)=E_{B}$, and $E_{C}(\theta)$ represents the effective capacity denoted as

$$
\begin{aligned}
E_{C}(\theta)= & \lim _{M \longrightarrow \infty} \\
& -\frac{1}{M \theta} \log \left(\mathbb{E}\left\{\exp \left(-\theta \sum_{n=0}^{M} R[n]\right)\right\}\right),
\end{aligned}
$$

where $\theta$ is a positive constant termed as QoS exponent and $\{R[n], n=0,1,2, \ldots\}$ is a discrete-time, stationary, and ergodic stochastic service process. $\mathbb{E}[\cdot]$ is the expectation operator. When the service processes are uncorrelated, the effective capacity can be expressed as

$$
E_{C}(\theta)=-\frac{1}{\theta} \log (\mathbb{E}\{\exp (-\theta R[n])\}) .
$$

Note that, for the secrecy system mentioned in this paper, if the channel gains during one frame satisfy $h_{l}>g_{l}$, the transmit data can be securely transferred at the nonzero secrecy rate. Thus, the secure effective capacity can be mathematically calculated as

$$
E_{s e c}(\theta)=-\frac{1}{\theta} \log \left(\mathbb{E}\left\{e^{-\theta R_{s e c}}\right\}\right) .
$$

Obviously, the secure effective capacity bounded by the minimal service rate is a monotonically decreasing function of $\theta$. Given the delay bound $D_{\max }$, the delay violation probability can be characterized by the QoS exponent $\theta$. A small $\theta$ corresponds to a loose violation probability requirement, while a large $\theta$ matches a strict QoS requirement. For multimedia applications, the secure effective capacity can be calculated with given $\theta$. Only if $E_{\text {sec }}(\theta) \geq E_{B}$ holds, the delay QoS guarantee can be satisfied.

\section{Resource Allocation Policy with Delay QoS Guarantee}

In this section, we investigate the joint power and time slot allocation policy to minimize the total cost of average power subjected to the given delay QoS constraint specified by the 
secure effective capacity of each user. The cost function is a $\beta$ fair cost function with a nonnegative parameter $\beta$ which can be employed to formulate the fair energy-efficient resource allocation and it is shown as

$$
V_{\beta}(\cdot)=\frac{(\cdot)^{1+\beta}}{(1+\beta)} .
$$

It is a convex and monotonically increasing function. The $\beta$-fairness is achieved by distributing the power to the user link that has consumed the smallest amount of power. The function achieves power minimization which seeks the most energy-efficient resource allocation with $\beta=0$ and min-max fairness with $\beta \longrightarrow \infty$ as two special cases.

To guarantee the QoS requirement of the secrecy system, the secure effective capacity with the corresponding delay QoS exponent should be no less than the effective bandwidth denoted as $\mathbf{E}:=\left[E_{1}, \ldots, E_{L}\right]^{T}$. Let $\overline{\mathbf{p}}:=\left[\bar{p}_{1}, \ldots, \bar{p}_{L}\right]^{T}$, $\boldsymbol{\tau}(\mathbf{h}, \mathbf{g}):=\left[\tau_{1}(\mathbf{h}, \mathbf{g}), \ldots, \tau_{L}(\mathbf{h}, \mathbf{g})\right]^{T}$, and $\mathbf{p}(\mathbf{h}, \mathbf{g}):=\left[p_{1}(\mathbf{h}, \mathbf{g})\right.$, $\left.\ldots, p_{L}(\mathbf{h}, \mathbf{g})\right]^{T}$ represent the average user power vector, the time fraction vector, and the power vector adapted to the CSI $\mathbf{h}$ and $\mathbf{g}$, respectively. Thus, we can formulate the optimization problem as

$$
\begin{aligned}
\min _{\overline{\mathbf{p}}, \boldsymbol{\tau}(\mathbf{h}, \mathbf{g}), \mathbf{p}(\mathbf{h}, \mathbf{g})} & \sum_{l=1}^{L} V_{\beta}\left(\bar{p}_{l}\right) \\
\text { s.t. } & \bar{p}_{l} \geq \mathbb{E}_{\mathbf{h}, \mathbf{g}}\left[p_{l}(\mathbf{h}, \mathbf{g})\right], \\
& -\frac{1}{\theta_{l}} \log \left\{\mathbb{E}_{\mathbf{h}, \mathbf{g}}\left[e^{-\theta_{l} r_{s e c}\left(\tau_{l}(\mathbf{h}, \mathbf{g}), p_{l}(\mathbf{h}, \mathbf{g})\right)}\right]\right\} \geq E_{l}, \\
& \sum_{l=1}^{L} \tau_{l}(\mathbf{h}, \mathbf{g}) \leq 1, \\
& \tau_{l}(\mathbf{h}, \mathbf{g}) \geq 0, p_{l}(\mathbf{h}, \mathbf{g}) \geq 0,
\end{aligned}
$$

where $\theta_{l}$ represents the QoS exponent of the $l$-th user and $l=$ $1, \ldots, L$. We find that the objective function in (9) is convex with respect to $\overline{p_{l}}$ and the second constraint can be converted into

$$
\mathbb{E}_{\mathbf{h}, \mathbf{g}}\left[e^{-\theta_{l} r_{s e c}\left(\tau_{l}(\mathbf{h}, \mathbf{g}), p_{l}(\mathbf{h}, \mathbf{g})\right)}\right] \leq e^{-\theta_{l} E_{l}}
$$

By evaluating the Hessian matrix $r_{s e c}\left(\tau_{l}(\mathbf{h}, \mathbf{g}), p_{l}(\mathbf{h}, \mathbf{g})\right)$ at $\tau_{l}(\mathbf{h}, \mathbf{g})$ and $p_{l}(\mathbf{h}, \mathbf{g})$, we can prove that $r_{s e c}\left(\tau_{l}(\mathbf{h}, \mathbf{g}), p_{l}(\mathbf{h}, \mathbf{g})\right)$ is a jointly concave function of $\tau_{l}(\mathbf{h}, \mathbf{g})$ and $p_{l}(\mathbf{h}, \mathbf{g})$. Since the expectation and exponent operations preserve the concavity, the constraint in (10) is also convex. In addition, the remaining constraints are all convex. Hence, (9) is a convex optimization problem with unique optimal solution.

3.1. Optimal Resource Allocation Algorithm. In this subsection, an optimal time slot and power allocation algorithm for the optimization problem (9) is proposed based on the Lagrangian dual technique in [28]. Let $\lambda:=\left[\lambda_{1}, \ldots, \lambda_{L}\right]^{T}$ and $\xi:=\left[\xi_{1}, \ldots, \xi_{L}\right]^{T}$ be the Lagrange multipliers associated with the average power constraint and the delay QoS constraint, respectively. For convenience, we set $\mathbf{X}:=\{\overline{\mathbf{p}}, \mathbf{p}(\cdot), \boldsymbol{\tau}(\cdot)\}, \Psi:=$ $\{\boldsymbol{\lambda}, \boldsymbol{\xi}\}$, and $\boldsymbol{Y}:=\{\mathbf{h}, \mathbf{g}\}$. Then, the average power constraint and delay QoS constraint are relaxed to form the Lagrangian function as

$$
\begin{aligned}
& L(\mathbf{X}, \Psi)=\sum_{l=1}^{L} V_{\beta}\left(\bar{p}_{l}\right)+\sum_{l=1}^{L} \lambda_{l}\left(\mathbb{E}_{\mathbf{Y}}\left[p_{l}(\mathbf{Y})\right]-\bar{p}_{l}\right)+\sum_{l=1}^{L} \xi_{l} \\
& \cdot\left(\mathbb{E}_{\mathbf{\Upsilon}}\left[e^{-\theta_{l} r_{s e c}\left(\tau_{l}(\mathbf{\Upsilon}), p_{l}(\mathbf{\Upsilon})\right)}\right]-e^{-\theta_{l} E_{l}}\right) \\
& =\sum_{l=1}^{L}\left(V_{\beta}\left(\bar{p}_{l}\right)-\lambda_{l} \bar{p}_{l}\right) \\
& +\sum_{l=1}^{L} \mathbb{E}_{\mathbf{\Upsilon}}\left[\lambda_{l} p_{l}(\mathbf{\Upsilon})+\xi_{l} \cdot e^{-\theta_{l} r_{s e c}\left(\tau_{l}(\Upsilon), p_{l}(\Upsilon)\right)}\right] \\
& -\sum_{l=1}^{L} \xi_{l} e^{-\theta_{l} E_{l}}
\end{aligned}
$$

The master dual function is further expressed as

$$
D(\Psi)= \begin{cases}\min _{\mathbf{X}} & L(\mathbf{X}, \boldsymbol{\Psi}) \\ \text { s.t. } & \sum_{l=1}^{L} \tau_{1}(\mathbf{h}, \mathbf{g}) \leq 1,\end{cases}
$$

and the dual problem of (9) can be expressed as $\max _{\Psi \succeq \mathbf{0}} D(\Psi)$. To find the optimal joint power and time slot allocation $\left(\overline{\mathbf{p}}^{*}, \boldsymbol{\tau}^{*}, \mathbf{p}^{*}\right)$ by solving the master dual function (12), we need to solve the decoupled subfunctions below:

$$
\min _{\bar{p}_{l}} \sum_{l=1}^{L}\left(V_{\beta}\left(\bar{p}_{l}\right)-\lambda_{l} \bar{p}_{l}\right),
$$

and

$$
\begin{gathered}
\min _{\mathbf{p}, \boldsymbol{\tau}} \sum_{l=1}^{L} \mathbb{E}_{\mathbf{\Upsilon}}\left[\lambda_{l} p_{l}(\mathbf{\Upsilon})+\xi_{l} \cdot e^{-\theta_{l} r_{s e c}\left(\tau_{l}(\mathbf{\Upsilon}), p_{l}(\mathbf{\Upsilon})\right)}\right] \\
-\sum_{l=1}^{L} \xi_{l} e^{-\theta_{l} E_{l}}
\end{gathered}
$$

$$
\text { s.t. } \quad \sum_{l=1}^{L} \tau_{1}(\Upsilon) \leq 1 .
$$

For each user $l$, (13) can be decoupled as

$$
\min _{\bar{p}_{l}} V_{\beta}\left(\bar{p}_{l}\right)-\lambda_{l} \bar{p}_{l}
$$

It is clear that solving (15) is equivalent to solve (13). For given $\lambda_{l}$, (15) is a deterministic convex optimization problem. Hence, we can readily solve it and get the optimal solution as

$$
\bar{p}_{l}^{*}(\Psi)=\lambda_{l}^{1 / \beta}
$$

For the subfunction (14) associated with $(\mathbf{p}, \boldsymbol{\tau})$, it can be decoupled as subfunctions under each channel realization 
like $\mathbf{h}$ and $\mathbf{g}$. Hence, the solution of (14) can be reduced to solve the following function:

$$
\begin{aligned}
& \min _{\mathbf{p}, \boldsymbol{\tau}} \sum_{l=1}^{L}\left[\lambda_{l} p_{l}(\mathbf{Y})+\xi_{l} \cdot e^{-\theta_{l} r_{s e c}\left(\tau_{l}(\Upsilon), p_{l}(\mathbf{Y})\right)}\right] \\
& \text { s.t. } \quad \sum_{l=1}^{L} \tau_{l}(\Upsilon) \leq 1 .
\end{aligned}
$$

It is easily proved that (17) is a convex optimization problem and its optimal solutions $p_{l}^{*}(\mathbf{Y}, \Psi)$ and $\tau_{l}^{*}(\mathbf{Y}, \Psi)$ can be obtained via the algorithms given in the following subsection. For $\forall l=1, \ldots, L$, the dual problem of $\max _{\Psi \geq 0} D(\Psi)$ can be solved by the subgradient iterative algorithm as

$$
\begin{aligned}
& \lambda_{l}[n+1]=\lambda_{l}[n]+\alpha[n]
\end{aligned}
$$

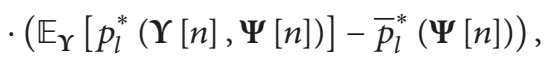

$$
\begin{aligned}
& \xi_{l}[n+1]=\xi_{l}[n]+\alpha[n] \\
& \cdot\left(\mathbb{E}_{\mathbf{\Upsilon}}\left[e^{-\theta_{l} r_{s e c}\left(\tau_{l}^{*}(\mathbf{Y}[n], \Psi[n]), p_{l}^{*}(\mathbf{\Upsilon}[n], \Psi[n])\right)}\right]-e^{-\theta_{l} E_{l}}\right),
\end{aligned}
$$

where $n$ represents the time index and $\alpha[n]$ is the step size.

3.2. Joint Power and Time Slot Allocation Policy for Each Channel Realization. In this subsection, we aim to investigate the convex optimization function (17) and propose an optimal joint power and time slot allocation policy for each channel realization with given $\lambda$ and $\boldsymbol{\xi}$. By relaxing the time slot constraint, the Lagrangian function becomes

$$
\begin{aligned}
& J\left(\tau_{l}(\Upsilon), p_{l}(\Upsilon)\right) \\
& =\sum_{l=1}^{L}\left[\lambda_{l} p_{l}(\mathbf{Y})+\xi_{l} \cdot e^{-\theta_{l} r_{s e c}\left(\tau_{l}(\mathbf{Y}), p_{l}(\mathbf{\Upsilon})\right)}\right]+\eta \\
& \cdot\left[\sum_{l=1}^{L} \tau_{l}(\Upsilon)-1\right]
\end{aligned}
$$

where $\eta$ denotes the Lagrangian multiplier associated with the time slot constraint. Then, we propose the optimal time slot and power assignment policy.

Define $p_{l}^{*}(\mathbf{Y}, \Psi)$ and $\tau_{l}^{*}(\mathbf{Y}, \Psi)$ as the desired optimal solutions. Applying the Karush-Kuhn-Tucker (KKT) conditions, for $\forall l=1, \ldots, L$, we can derive the following necessary and sufficient conditions on $p_{l}^{*}(\mathbf{Y}, \Psi)$ and $\tau_{l}^{*}(\Upsilon, \Psi)$ as

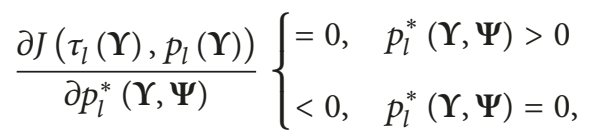

$$
\begin{aligned}
& \frac{\partial J\left(\tau_{l}(\mathbf{Y}), p_{l}(\mathbf{Y})\right)}{\partial \tau_{l}^{*}(\mathbf{Y}, \Psi)} \begin{cases}>0, & \tau_{l}^{*}(\mathbf{Y}, \Psi)=0 \\
=0, & 0<\tau_{l}^{*}(\mathbf{Y}, \Psi)<1 \\
<0, & \tau_{l}^{*}(\mathbf{Y}, \Psi)=1 .\end{cases}
\end{aligned}
$$

Equation (21) means that the derivative at the minimum point is zero if the minimum lies within the constraint region $(0,1)$. If the minimum is located on the boundary of the constraint region, the derivative is either positive or negative pointing towards the interior of the constraint region along all directions. With the above two optimality conditions, we can calculate the optimal power and time slot allocation arithmetically. From (20), we can obtain

$$
\begin{aligned}
\lambda_{l}- & \frac{\xi_{l} \theta_{l}}{\log 2} \cdot e^{-\theta_{l} \tau_{l}(\mathbf{\Upsilon}) \log _{2}\left(\left(1+h_{l} p_{l}(\mathbf{\Upsilon}) / \tau_{l}(\mathbf{\Upsilon})\right) /\left(1+g_{l} p_{l} / \tau_{l}(\mathbf{\Upsilon})\right)\right)} \\
& \cdot \frac{h_{l}-g_{l}}{\left(1+h_{l} p_{l}(\mathbf{\Upsilon}) / \tau_{l}(\mathbf{\Upsilon})\right)\left(1+g_{l} p_{l}(\mathbf{\Upsilon}) / \tau_{l}(\mathbf{\Upsilon})\right)} \\
= & 0 .
\end{aligned}
$$

Let $\left\{\tau_{l}^{*}(\mathbf{Y}, \Psi), \forall l\right\}$ be the optimal time slot assignment; then the corresponding power allocation $p_{l}^{*}(\Upsilon, \Psi)$ satisfies the following formula:

$$
\begin{gathered}
\frac{\left(1+g_{l} p_{l}^{*}(\mathbf{\Upsilon}, \boldsymbol{\Psi}) / \tau_{l}^{*}(\mathbf{\Upsilon}, \boldsymbol{\Psi})\right)^{B-1}}{\left(1+h_{l} p^{*}(\mathbf{Y}, \boldsymbol{\Psi}) / \tau_{l}^{*}(\mathbf{Y}, \boldsymbol{\Psi})\right)^{B+1}}=\frac{\gamma_{0}}{h_{l}-g_{l}}, \\
\gamma_{0}<h_{l}-g_{l}, \tau_{l}^{*}(\mathbf{\Upsilon}, \boldsymbol{\Psi})>0, \\
p_{l}^{*}(\mathbf{\Upsilon}, \boldsymbol{\Psi})=0, \\
\gamma_{0} \geq h_{l}-g_{l}, \tau_{l}^{*}(\mathbf{Y}, \boldsymbol{\Psi})=0,
\end{gathered}
$$

with $B \triangleq \theta_{l} \tau_{l}^{*}(\mathbf{Y}, \Psi) / \log (2)$ and $\gamma_{0} \triangleq\left(\lambda_{l} \cdot \log (2)\right) / \xi_{l} \theta_{l}$. It is observed that the power allocation of the active user has the same form as that for the single-user QoS-driven secrecy system [25, eq.(15)] with different cutoff thresholds at each channel realization. Furthermore, we analyze the time slot allocation policy which follows Lemma 1.

Lemma 1. Assume that, for each channel realization, i.e., $\mathbf{h}$ and $\mathbf{g}$; in the context of secure transmission, there exists at most one user allocated with a strictly positive power and we assert it the "winner-takes-all" policy.

The users in the system whose channel gains may not satisfy the secrecy communication condition $h_{l}>g_{l}$ will not participate in the time slot allocation; i.e., $\tau_{l}^{*}(\mathbf{Y}, \Psi)=0$ for $h_{l}<g_{l}$. The following derivations are all satisfied with $h_{l}>g_{l}$. Taking the partial derivative of (19) with respect to $\tau_{l}(\Upsilon)$, we have

$$
\begin{aligned}
& \frac{\partial J\left(\tau_{l}(\mathbf{\Upsilon}), p_{l}(\mathbf{\Upsilon})\right)}{\partial \tau_{l}(\mathbf{Y})}=\eta-\xi_{l} \theta_{l} \\
& \cdot e^{-\theta_{l} \tau_{l}(\mathbf{\Upsilon}) \log _{2}\left(\left(\tau_{l}(\mathbf{\Upsilon})+h_{l} p_{l}(\mathbf{\Upsilon})\right) /\left(\tau_{l}(\mathbf{\Upsilon})+g_{l} p_{l}(\mathbf{\Upsilon})\right)\right)} \\
& \quad \cdot\left[\log _{2} \frac{1+h_{l} p_{l}(\mathbf{\Upsilon}) / \tau_{l}(\mathbf{\Upsilon})}{1+g_{l} p_{l}(\mathbf{\Upsilon}) / \tau_{l}(\mathbf{\Upsilon})}+\frac{1}{\log 2}\right. \\
& \left.\cdot \frac{\left(g_{l}-h_{l}\right) \cdot p_{l}(\mathbf{\Upsilon})}{\left(\tau_{l}(\mathbf{Y})+h_{l} p_{l}(\mathbf{\Upsilon})\right)\left(\tau_{l}(\mathbf{\Upsilon})+g_{l} p_{l}(\mathbf{\Upsilon})\right)}\right] .
\end{aligned}
$$


Here, we substitute $p_{l}^{*}(\mathbf{Y}, \Psi)$ obtained by (23) into (24) and simplify it with (22). According to the KKT condition (21), for $\forall l=1, \ldots, L$, we can get

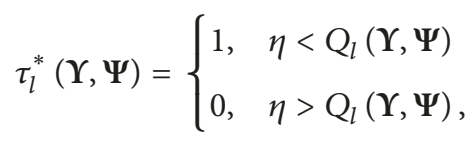

where $Q_{l}(\mathbf{Y}, \Psi)$ is termed as the quality function and written as

$$
\begin{aligned}
& Q_{l}(\mathbf{Y}, \Psi)=\lambda_{l} \\
& \cdot \log _{2} \frac{\left(1+h_{l} p_{l}^{*}(\mathbf{Y}, \Psi)\right)\left(1+g_{l} p_{l}^{*}(\mathbf{Y}, \Psi)\right)}{h_{l}-g_{l}} \\
& \cdot \log _{2} \frac{1+h_{l} p_{l}^{*}(\mathbf{Y}, \Psi)}{1+g_{l} p_{l}^{*}(\mathbf{Y}, \Psi)}-\lambda_{l} p_{l}^{*}(\mathbf{Y}, \Psi) .
\end{aligned}
$$

Since the constraint must be satisfied, only the user whose $Q_{l}(\mathbf{Y}, \Psi)$ is the smallest can occupy the whole time slot. In detail, we write

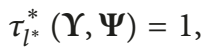

$$
\begin{aligned}
& \tau_{l}^{*}(\mathbf{Y}, \Psi)=0, \\
& l \neq l^{*},
\end{aligned}
$$

with

$$
l^{*}=\underset{l}{\arg \max } Q_{l}(\mathbf{Y}, \Psi) .
$$

Given a time slot, there will be a set $\left\{Q_{1}(\Upsilon, \Psi), Q_{2}(\Upsilon, \Psi)\right.$, $\left.\ldots, Q_{L}(\Upsilon, \Psi)\right\}$ with $L$ elements related to different corresponding users. The user $l$ whose $Q_{l}(Y, \Psi)$ is the minimum in the set will occupy the whole time slot. That is to say, we can easily derive the time slot allocation scheme with the obtained set $\left\{Q_{1}(\Upsilon, \Psi), Q_{2}(\Upsilon, \Psi), \ldots, Q_{L}(\Upsilon, \Psi)\right\}$.

3.3. Stochastic Joint Time Slot and Power Allocation Algorithm. According to Lagrangian duality theory [30], the strong duality of the primary problem (9) should hold, which guarantees the solution of the primary problem through the dual problem $\max _{\Psi \geq 0} D(\Psi)$. To solve this dual problem, we need the explicit knowledge of the CDF of the fading channel in order to evaluate the expected values $\mathbb{E}_{\Upsilon}[\cdot]$, whereas for some practical wireless communication environments, it is impractical or impossible to obtain the CDF of the fading channels. Thus, the joint power and time slot allocation algorithm which can operate without the knowledge of the channel CDF and approach the optimal strategy by learning the channel statistics should be achieved urgently. The above issue can be solved via the utilization of the stochastic optimization theory in [34]. Note that $D(\Psi)$ is a convex function of $\Psi$; thus $\max _{\Psi \succeq 0} D(\Psi)$ is a stochastic convex optimization problem. Hence, employing the stochastic subgradient iterative method [35] with unknown channel CDF, we can get a stochastic subgradient iteration algorithm based on the channel realizations $(\mathbf{h}[n]$ and $\mathbf{g}[n])$ at time slot $n$ for $\forall l=1, \ldots, L$ as

$$
\begin{aligned}
& \widehat{\lambda}_{l}[n+1]=\widehat{\lambda}_{l}[n] \\
& \quad+\alpha[n]\left(p_{l}^{*}(\mathbf{\Upsilon}[n], \widehat{\Psi}[n])-\bar{p}_{l}^{*}(\widehat{\Psi}[n])\right), \\
& \widehat{\xi}_{l}[n+1]=\widehat{\xi}_{l}[n] \\
& \quad+\alpha[n]\left(\left[e^{-\theta_{l} r_{s e c}\left(\tau_{l}^{*}(\mathbf{\Upsilon}[n], \widehat{\Psi}[n]), p_{l}^{*}(\mathbf{\Upsilon}[n], \widehat{\Psi}[n])\right)}\right]-e^{-\theta_{l} E_{l}}\right),
\end{aligned}
$$

where ${ }^{\wedge}$ denotes the stochastic estimation, compared with (18). Equations (29) and (18) correspond to the primary and averaged systems, respectively. We can prove the convergence of the stochastic subgradient iteration by employing the stochastic locking theorem in [36]. The stochastic locking theorem holds only if certain regularity conditions are satisfied, such as the stochastic Lipschitz conditions for system perturbations. According to [37], we have the following Lemma 2 which confirms that these regularity conditions are satisfied for the primary and average systems with TDMA scheme, provided that the continuous channel CDF can be obtained.

Lemma 2. For ergodic fading channel with continuous CDF, if the primary system (18) and its averaged system (29) are both initialized with $\widehat{\Psi}[0] \equiv \Psi[0]$, then, for any time interval T, it holds that

$$
\max _{1 \leq n \leq T / c}\|\widehat{\Psi}[n]-\Psi[n]\| \leq c_{T}(c)
$$

with possibility 1 ,

with $c_{T}(c) \longrightarrow 0$ as $c \longrightarrow 0$.

Trajectory locking is the key of the asymptotic optimality of the stochastic iterations. Lemma 2 rigorously establishes that the trajectories of the primary and average systems, corresponding to (29) and (18), respectively, remain close to each other over any time interval $T$ for small enough step size $c$. That is to say, the distance of the two trajectories is bounded in probability by a constant $c_{T}(c)$ which will vanish as $c \longrightarrow 0$. Hence, the stochastic power and time slot allocation scheme will approach the global optimal solution of (11). The detailed theoretical analysis of the stochastic optimization algorithm is a complex and tedious work and it is not the main purpose of our paper. In addition, for any stochastic approximation scheme, the Lagrange multipliers in (19) converge to the optimal values or hover within a small neighborhood around the optimal values with the size proportional to the step size c.

\section{Non-Delayed Resource Allocation Policy}

The optimal resource allocation which satisfies the delay QoS constraint has been investigated above. In this section, we also present a non-delayed resource allocation scheme without considering the delay QoS requirement for comparison. When the delay QoS constraint of the optimization problem 
(9) becomes the secrecy rate constraint, the jointly power and time slot allocation $\left(p_{l}^{+}, \tau_{l}^{+}\right)$will obey the following equations:

$$
\begin{aligned}
& p_{l}^{+}= \begin{cases}\frac{1}{2}\left(\sqrt{\left(\frac{1}{h_{l}}-\frac{1}{g_{l}}\right)^{2}+\frac{4}{v_{l}}\left(\frac{1}{g_{l}}-\frac{1}{h_{l}}\right)}-\left(\frac{1}{h_{l}}+\frac{1}{g_{l}}\right)\right), & v_{l}<h_{l}-g_{l}, \tau_{l}^{+}>0 \\
0, & \text { else }\end{cases} \\
& \tau_{l^{+}}^{+}=1, \\
& \tau_{l}^{+}=0,
\end{aligned}
$$

$$
l \neq l^{+}, l^{+}=\underset{l}{\arg \min }\left\{\zeta_{l} p_{l}^{+}-\varepsilon_{l}\left[\log _{2}\left(1+h_{l} p_{l}^{+}\right)-\left(1+h_{l} p_{l}^{+}\right)\right]\right\},
$$

where $\zeta_{l}$ and $\varepsilon_{l}$ are the Lagrangian multipliers associated with the average power constraint and the secrecy rate constraint for the $l$-th user, respectively, and $\nu_{l} \triangleq \zeta_{l} \tau_{l}^{+} \log 2 / \varepsilon_{l}$.

In fact, for a given time slot, the power allocation algorithm (31) is the same as the scheme [33, eq.(7)] of the single-user secrecy system. Besides, considering the power allocation scheme (23) which supports the QoS requirement with $\theta \longrightarrow 0$, the optimal power allocation factor $p_{l}^{*}$ converges to (31). This phenomenon illustrates that the optimal QoS-driven resource allocation policy merges with the non-delayed policy when we relax the QoS requirement.

\section{Numerical Results and Analysis}

In this section, the numerical results of the proposed optimal resource allocation policy and non-delayed policy are provided to verify the foregoing theoretical analysis. We assume that the TDMA secrecy networks include three transmit users, one legitimate receiver, and one eavesdropper. The fading channels of different users are independent to each other. Besides, the channel power gains of the main user channels $h_{l}$ and the wiretap channels $g_{l}$ obey Rayleigh fading distributions with variance $\bar{h}_{l}$ and $\bar{g}_{l}$, respectively, and $h_{l}>$ $g_{l}(l=1,2,3)$. For simplicity, we assume that all the links suffer from the same delay impact. Specific system parameters are given in the corresponding figures.

The convergence performance of the Lagrange multipliers of the proposed optimal resource allocation policy is shown in Figure 2. In the simulation, six Lagrange multipliers, $\left(\lambda_{1}, \lambda_{2}, \lambda_{3}\right)$ and $\left(\xi_{1}, \xi_{2}, \xi_{3}\right)$, which are associated, respectively, with the average power constraint and the delay QoS constraint, are chosen arbitrarily to make the example. It can be observed from Figure 2 that all the six multipliers become stable after 12 iterations. Thus, we can get the same conclusion as what we have discussed in the end of Section 3 that the convergence of the proposed optimal policy can be guaranteed.

Figure 3 shows the power consumption of the user links $1-3$ which adopt the optimal policy under different $\beta$ fairness $(\beta=0.01,3,6)$. In addition, two comparative schemes, non-delayed scheme and fixed-access scheme, are also included for $\beta=0.01$. Here, the average normalized channel power gains for different user links are set to be $\bar{h}_{1}=3, \bar{h}_{2}=0, \bar{h}_{3}=-3, \bar{g}_{1}=\bar{g}_{2}=\bar{g}_{1}=-3 \mathrm{dBW}$, and the QoS exponents for the user links $1-3$ are $\theta_{1}=\theta_{2}=\theta_{3}=1$. From the individual average power in Figure 3, we observe that, for smaller $\beta$ such as $\beta=0.01$, the power gaps between different users are apparent and the resource allocation scheme assigns more power to the worst channel (link 3), which illustrates that we can increase the transmit power to maintain the system throughput if the channel becomes worse. It is also seen that the power consumed by different user links gets close to each other when $\beta$ becomes larger. Meanwhile, the total average power increases if $\beta$ increases. Hence, we can draw the conclusion that the fairness can be improved by consuming much more total power.

For comparison, Figure 3 also presents the total average power consumption of the fixed-access scheme and the nondelayed scheme. The fixed-access scheme assigns equal time fractions $\left(\tau_{1}=\tau_{2}=\tau_{3}=1 / 3\right)$ to the three links and each link still adopts the optimal power allocation strategy. We notice that much more total power is required for the fixed-access scheme than that of the optimal policy due to the neglect of the multiuser diversity and fairness. Since the non-delayed policy does not consider the delay QoS requirement during the joint power and time slot allocation, more total power is also needed to achieve the delay QoS guarantee. In a word, the proposed optimal policy can save much more power and the $\beta$-fair cost function can balance the energy efficiency and the fairness well.

In Figures 4 and 5, we plot the total average transmit power as a function of the delay QoS exponent under different resource allocation policies. We consider two cases with different eavesdropper conditions. The total average transmit power with better eavesdropper channel condition is shown in Figure 4. Figure 5 shows the total average transmit power with worse eavesdropper channel condition. The average main user channel gains are assumed to be $\bar{h}_{1}=3$, $\bar{h}_{2}=0$, and $\bar{h}_{3}=-3$ for the two conditions. We can observe from Figures 4 and 5 that the total average transmit power increases if the delay QoS exponent increases. As 


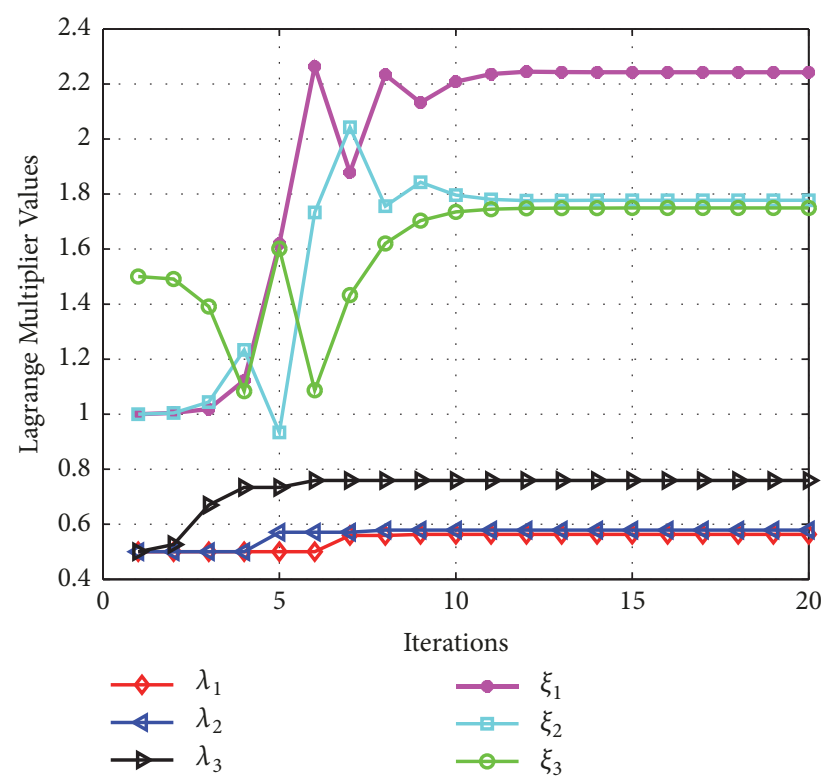

FIGURE 2: Convergence of Lagrange multipliers.
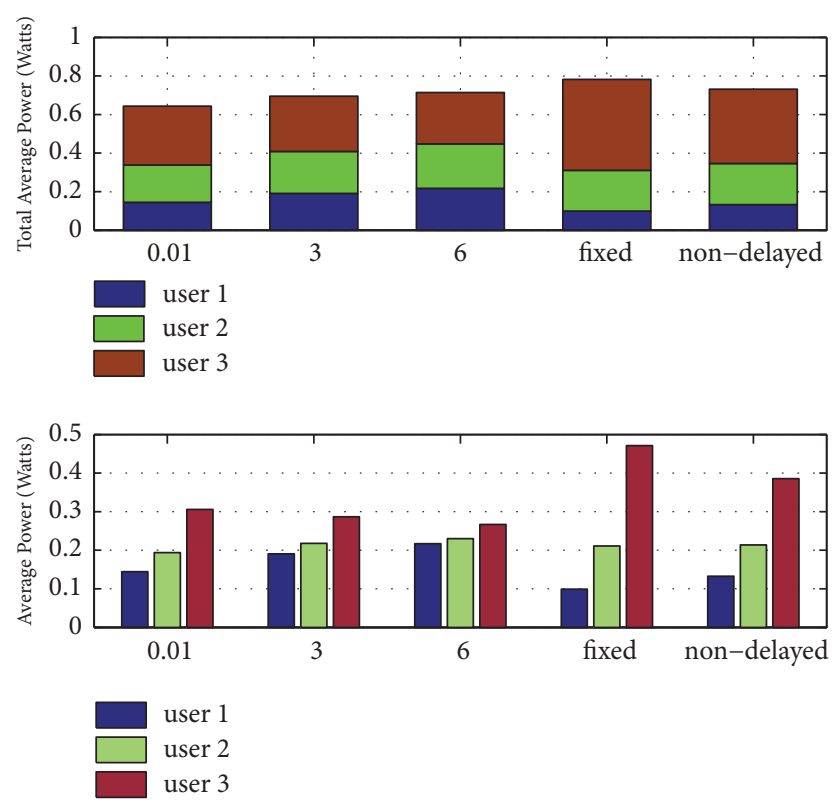

FIGURE 3: Total average power and individual average power.

expected, the proposed optimal resource allocation policy commonly consumes the minimal total average transmit power compared with the non-delayed policy and the fixedaccess policy. In addition, the optimal resource allocation policy converges to the non-delayed policy under a relatively small QoS exponent, which is coherent with the theoretical analysis above. Furthermore, by comparing the two figures, we find that the gap between the optimal policy and the nondelayed policy becomes significant when the eavesdropper channel becomes worse, which shows the advantages of the proposed optimal allocation policy under the worse eavesdropper channel condition.

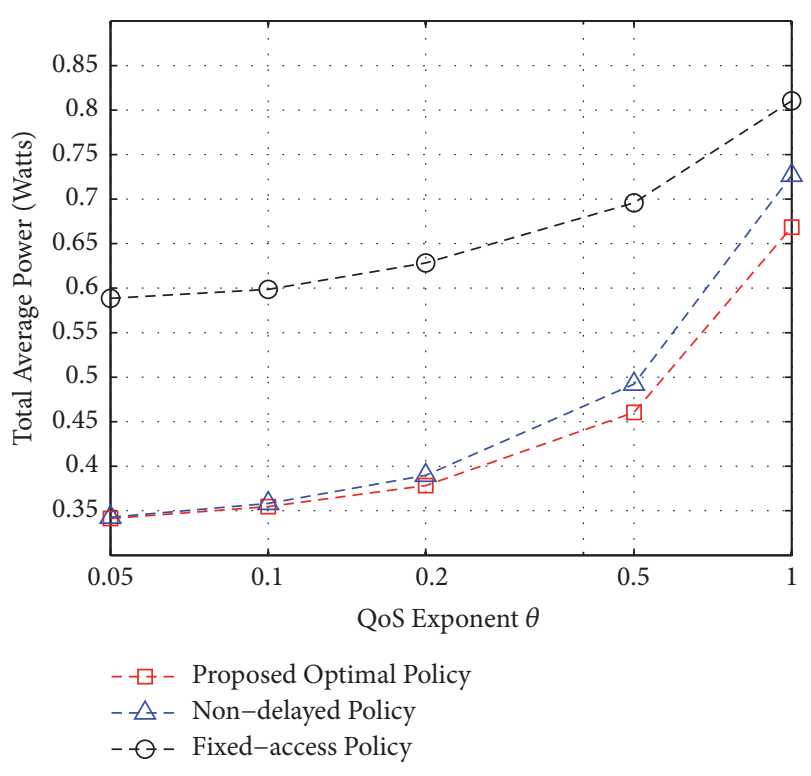

FIGURE 4: Total average power versus delay QoS exponent with wiretap channel conditions $\bar{g}_{1}=\bar{g}_{2}=\bar{g}_{3}=-3 \mathrm{dBW}$.

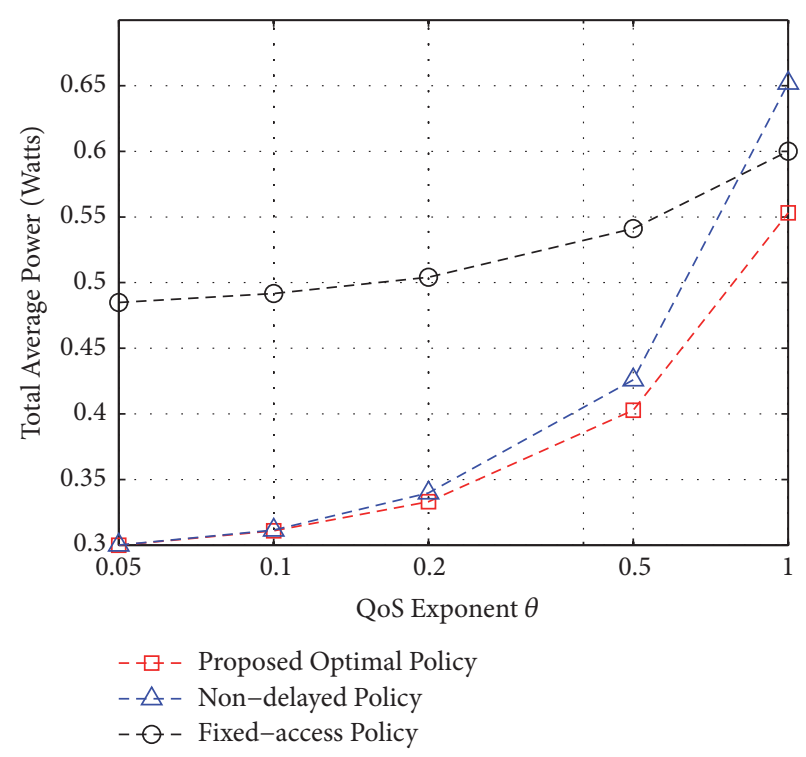

FIGURE 5: Total average power versus delay QoS exponent with wiretap channel conditions $\bar{g}_{1}=\bar{g}_{2}=\bar{g}_{3}=-20 \mathrm{dBW}$.

We provide the performance of the total average power versus the violation probability with delay bound $D_{\max }$ in Figure 6. In the simulation, we set the delay boundary as $D_{\max }=20 \mathrm{~ms}$. Given the delay boundary $D_{\max }$, the violation probability will arise if the delay requirement $\theta$ decreases according to (4). Under a certain violation probability, the proposed optimal resource allocation policy can achieve the minimum total average power among the three policies. Furthermore, it can be observed from Figure 6 that the total average transmit power will decrease when the violation probability increases. That is to say, a smaller delay requirement will lead to a lower total average power, which 


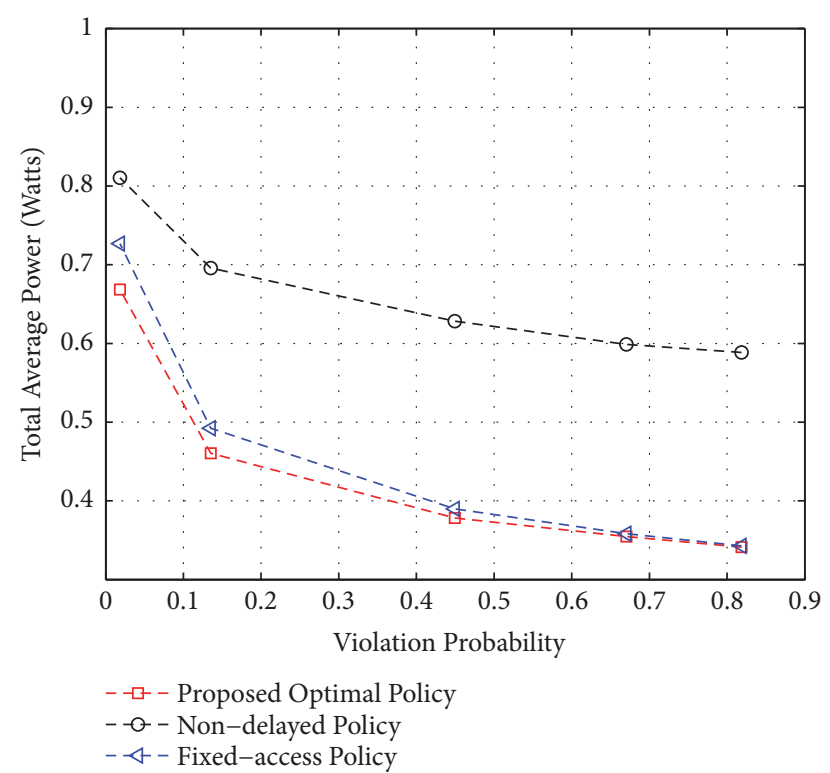

FIGURE 6: Total average power versus violation probability with delay boundary $D_{\max }=20 \mathrm{~ms}$.

is in agreement with our theoretical analysis above and also matches the results in Figure 5.

\section{Conclusions}

In this paper, we propose a fair energy-efficient resource allocation policy for the multiuser TDMA secrecy system. The system model is established for the secure transmission in the presence of an eavesdropper. By employing the secure effective capacity and $\beta$-fair cost function, we analytically study the joint power and time slot allocation policy which minimizes the $\beta$-fair cost function of the average transmit power while fulfilling the individual delay QoS requirement. A subgradient iterative algorithm is employed to solve the optimal resource allocation problem based on the convex optimization theory. In addition, the stochastic optimization is utilized to solve the problem. The limits of the total average power with the delay QoS constraint are derived based on the analytical results. The proposed optimal resource allocation scheme exhibits excellent energy efficiency compared with the suboptimal non-delayed scheme and the fixed-access scheme, especially in the case of stringent delay QoS requirement and worse eavesdropper channel condition. It also illustrates that the proposed stochastic iterative algorithm can approach the optimal strategy.

\section{Data Availability}

The data used to support the findings of this study are available from the corresponding author upon request.

\section{Conflicts of Interest}

The authors declare that there are no conflicts of interest regarding the publication of this paper.

\section{Acknowledgments}

This work was supported in part by International Science \& Technology Cooperation Program of China (2015DFG12580), National Science Foundation of China (61771291 and 61571272), Key R\&D Program of Shandong Province (2018GGX101009), the Fundamental Research Funds of Shandong University (2016JC010), and the Ministry of Science, ICT (MSIT), South Korea, supervised by the IITP through the ITRC support program (IITP-2018-2014-100729).

\section{References}

[1] C. E. Shannon, "Communication theory of secrecy systems," Bell Labs Technical Journal, vol. 28, pp. 656-715, 1949.

[2] A. D. Wyner, “The wire-tap channel," Bell Labs Technical Journal, vol. 54, no. 8, pp. 1355-1387, 1975.

[3] I. Csiszar and J. Korner, "Broadcast channels with confidential messages," IEEE Transactions on Information Theory, vol. 24, no. 3, pp. 339-348, 1978.

[4] M. Hellman, "The Gaussian wire-tap channel," IEEE Transactions on Information Theory, vol. 24, no. 4, pp. 451-456, 1978.

[5] A. Khisti and G. W. Wornell, "Secure transmission with multiple antennas I: the MISOME wiretap channel," IEEE Transactions on Information Theory, vol. 56, no. 7, pp. 3088-3104, 2010.

[6] X. Guan, Y. Cai, Y. Wang, and W. Yang, "Increasing secrecy capacity via joint design of cooperative beamforming and jamming," in Proceedings of the IEEE 22nd International Symposium on Personal, Indoor and Mobile Radio Communications (PIMRC '11), pp. 1279-1283, Toronto, Canada, September 2011.

[7] K. Cumanan, Z. Ding, B. Sharif, G. Y. Tian, and K. K. Leung, "Secrecy rate optimizations for a MIMO secrecy channel with a multiple-antenna eavesdropper," IEEE Transactions on Vehicular Technology, vol. 63, no. 4, pp. 1678-1690, 2014.

[8] Z. Chu, K. Cumanan, Z. Ding, M. Johnston, and S. Y. Le Goff, "Secrecy rate optimizations for a MIMO secrecy channel with a cooperative jammer," IEEE Transactions on Vehicular Technology, vol. 64, no. 5, pp. 1833-1847, 2015.

[9] E. A. Jorswieck and A. Wolf, "Resource allocation for the wire-tap multi-carrier broadcast channel," in Proceedings of the International Conference on Telecommunications, pp. 1-6, June 2008.

[10] Z. Li, R. Yates, and W. Trappe, "Secrecy capacity of independent parallel channels," in Proceedings of the 44th Annual Allerton Conference on Communication, Control, and Computing, pp. 841-848, September 2006.

[11] X. Wang, M. Tao, J. Mo, and Y. Xu, "Power and subcarrier allocation for physical-layer security in ofdma networks," in Proceedings of the IEEE International Conference on Communications (ICC '11), pp. 1-5, Kyoto, Japan, June 2011.

[12] H. Qin, X. Chen, X. Zhong, F. He, M. Zhao, and J. Wang, "Joint power allocation and artificial noise design for multiuser wiretap OFDM channels," in Proceedings of the IEEE International Conference on Communications (ICC '13), pp. 786-791, Budapest, Hungary, June 2013.

[13] A. Jindal and R. Bose, "Resource allocation for secure multicarrier AF relay system under total power constraint," IEEE Communications Letters, vol. 19, no. 2, pp. 231-234, 2015. 
[14] J. Huang and A. L. Swindlehurst, "Cooperative jamming for secure communications in MIMO relay networks," IEEE Transactions on Signal Processing, vol. 59, no. 10, pp. 4871-4884, 2011.

[15] J. Chen, X. Chen, T. Liu, and L. Lei, "Energy-efficient power allocation for secure communications in large-scale MIMO relaying systems," in Proceedings of the IEEE/CIC International Conference on Communications in China (ICCC '14), pp. 385390, October 2014.

[16] H. Zhang, C. Jiang, N. C. Beaulieu, X. Chu, X. Wang, and T. Q. S. Quek, "Resource allocation for cognitive small cell networks: a cooperative bargaining game theoretic approach," IEEE Transactions on Wireless Communications, vol. 14, no. 6, pp. 3481-3493, 2015.

[17] N. Mokari, S. Parsaeefard, H. Saeedi, and P. Azmi, "Cooperative secure resource allocation in cognitive radio networks with guaranteed secrecy rate for primary users," IEEE Transactions on Wireless Communications, vol. 13, no. 2, pp. 1058-1073, 2014.

[18] N. Mokari, S. Parsaeefard, H. Saeedi, P. Azmi, and E. Hossain, "Secure robust ergodic uplink resource allocation in relayassisted cognitive radio networks," IEEE Transactions on Signal Processing, vol. 63, no. 2, pp. 291-304, 2015.

[19] T. Wang, L. Song, Z. Han, X. Cheng, and B. Jiao, "Power allocation using Vickrey auction and sequential first-price auction games for physical layer security in cognitive relay networks," in Proceedings of the IEEE International Conference on Communications (ICC '12), pp. 1683-1687, June 2012.

[20] H. Zhang, T. Wang, L. Song, and Z. Han, "Radio resource allocation for physical-layer security in D2D underlay communications," in Proceedings of the 1st IEEE International Conference on Communications (ICC '14), pp. 2319-2324, June 2014.

[21] F. Chiti, D. D. Giacomo, R. Fantacci, and L. Pierucci, "Interference aware approach for D2D communications," in Proceedings of the IEEE International Conference on Communications (ICC '16), pp. 1-6, June 2016.

[22] P. Gandotra, R. K. Jha, and S. Jain, "A survey on device-todevice (D2D) communication: architecture and security issues," Journal of Network and Computer Applications, vol. 78, pp. 9-29, 2017.

[23] D. Wu, Providing quality-of-service guarantees in wireless networks [Ph.D. dissertation], Carnegie Mellon University, 2003.

[24] D. Wu and R. Negi, "Effective capacity: a wireless link model for support of quality of service," IEEE Transactions on Wireless Communications, vol. 2, no. 4, pp. 630-643, 2003.

[25] T. Wang, P. Ma, and Y. Ma, "Adaptive power allocation with quality-of-service guarantees in secure wireless communications," in Proceedings of the 9th International Conference on Communications and Networking in China (CHINACOM '14), pp. 508-512, Maoming, China, August 2014.

[26] Q. Pei, H. Li, H. Zhao, N. Li, and Y. Min, "Security in cognitive radio networks," Journal of Communication, no. 1, pp. 144-158, 2013.

[27] X. Wang, D. Wang, H. Zhuang, and S. D. Morgera, "Fair energyefficient resource allocation in wireless sensor networks over fading TDMA channels," IEEE Journal on Selected Areas in Communications, vol. 28, no. 7, pp. 1063-1072, 2010.

[28] S. Boyd and L. Vandenberghe, Convex Optimization, Cambridge University Press, 2004.

[29] D. P. Bertsekas, Nonlinear Programming, Athena Scientific, Belmont, Mass, USA, 2nd edition, 1999.

[30] D. Palomar and M. Chiang, "A tutorial on decomposition methods and distributed network resource allocation," IEEE
Journal on Selected Areas in Communications, vol. 24, no. 8, pp. 1439-1451, 2006.

[31] M. Chiang, S. H. Low, A. R. Calderbank, and J. C. Doyle, "Layering as optimization decomposition: a mathematical theory of network architectures," Proceedings of the IEEE, vol. 95, no. 1, pp. 255-312, 2007.

[32] S. L. Y. Cheong and M. E. Hellman, "The Gaussian wire-tap channel," IEEE Transactions on Information Theory, vol. 24, no. 4, pp. 451-456, 1978.

[33] P. K. Gopala, L. Lai, and H. E. Gamal, "On the secrecy capacity of fading channels," IEEE Transactions on Information Theory, vol. 54, no. 10, pp. 4687-4698, 2008.

[34] P. Kall and S. W. Wallace, Stochastic Programming, John Wiley \& Sons, Chichester, UK, 1994.

[35] R. T. Rockafellar and R. J. Wets, "Stochastic convex programming: basic duality," Pacific Journal of Mathematics, vol. 62, no. 1, pp. 173-195, 1976.

[36] V. Solo and X. Kong, Adaptive Signal Processing Algorithms: Stability and Performance, Prentice Hall, 1995.

[37] X. Wang and G. B. Giannakis, "Resource allocation for wireless multiuser OFDM networks," IEEE Transactions on Information Theory, vol. 57, no. 7, pp. 4359-4372, 2011. 


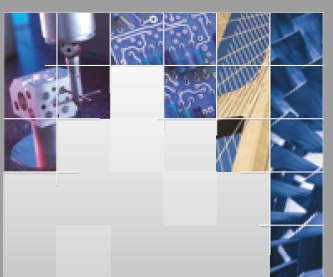

\section{Enfincering}
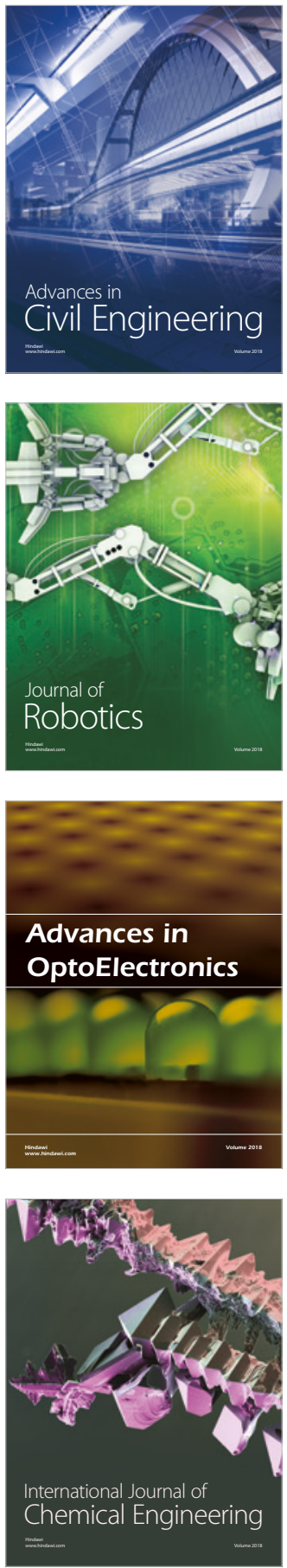

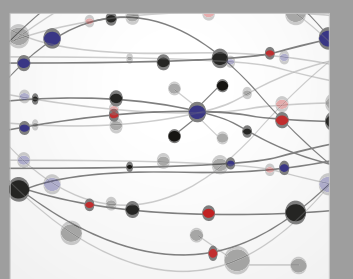

\section{Rotating \\ Machinery}

The Scientific World Journal

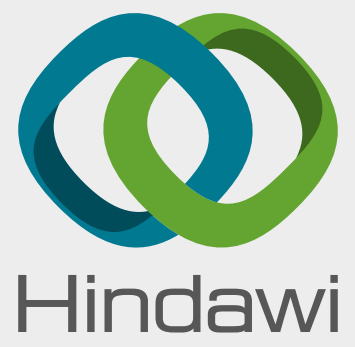

Submit your manuscripts at

www.hindawi.com
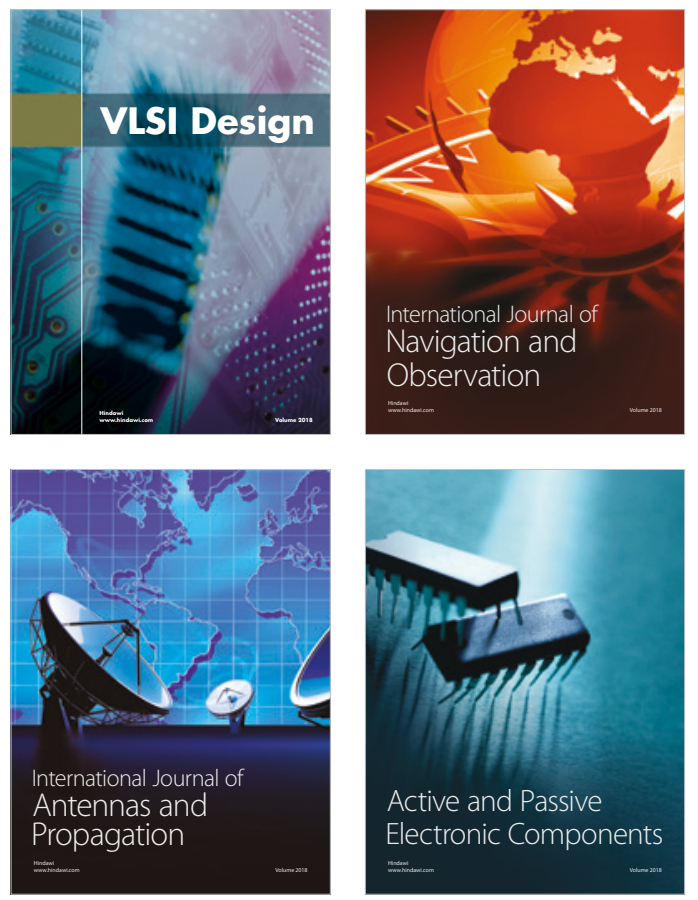
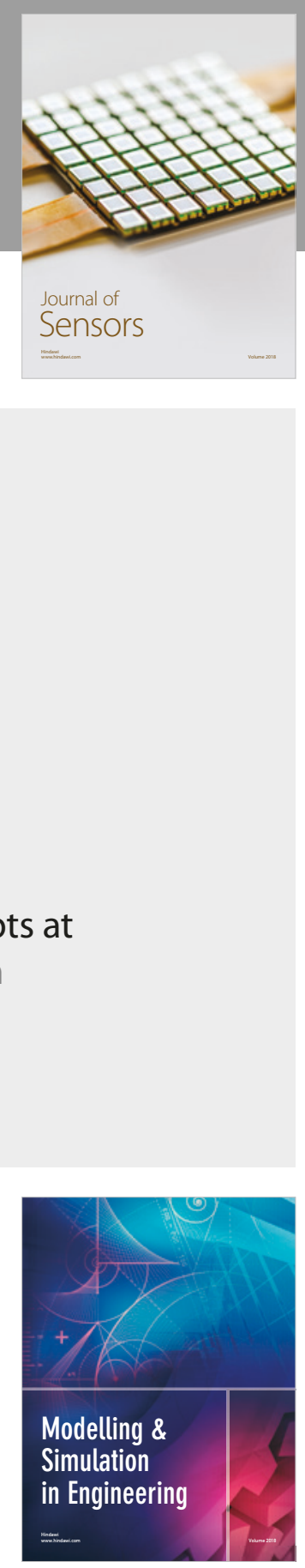

\section{Advances \\ Multimedia}
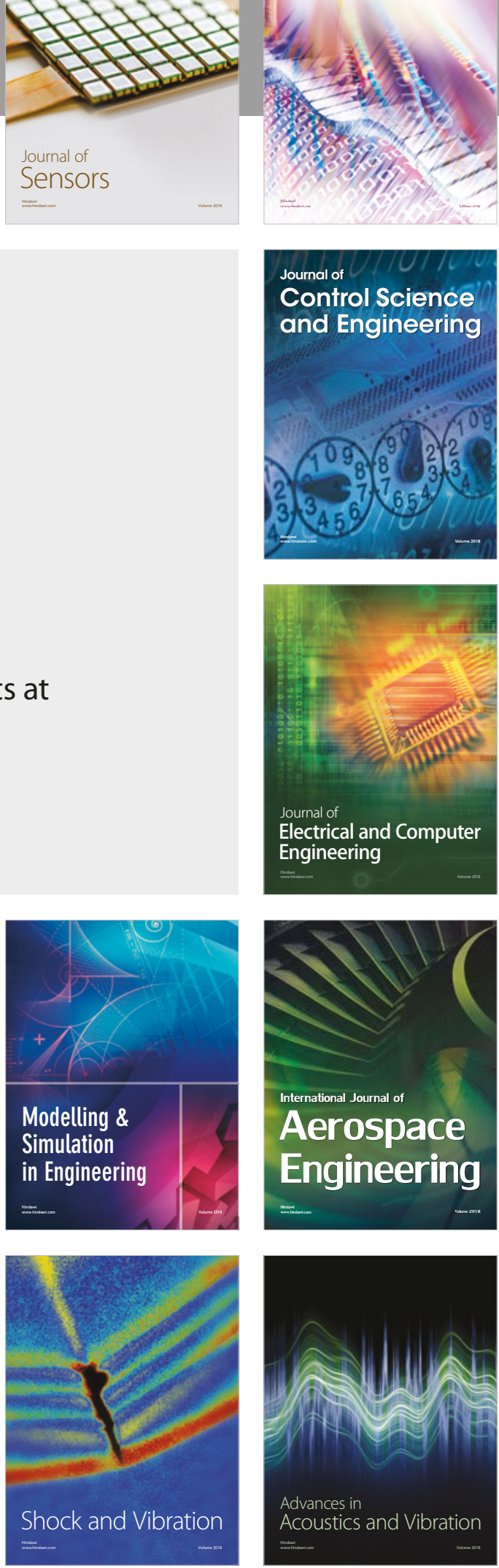